\title{
Effect of aquatic exercise on gait in persons with chronic stroke: a meta-analysis study in Korea
}

\author{
Dong-Jin Lee ${ }^{a}$, Sung-Hyoun Cho ${ }^{b}$ \\ a'Department of Physical Therapy, Gwangju Health University, Gwangju, Republic of Korea \\ ${ }^{b}$ Department of Physical Therapy, Nambu University, Gwangju, Republic of Korea
}

Objective: Based on the results of previous studies, it is necessary to analyze gait and discuss and present the effects of aquatic exercise for chronic stroke. The purpose of this study was to present objective data on the effect of aquatic exercise on the gait of persons with stroke by performing a meta-analysis.

Design: A systematic review and meta-analysis.

Methods: We performed a meta-analysis of 23 studies that investigated the effects of aquatic exercise performed between 2006 and 2017. The studies were searched on the basis of the participants, intervention, comparison, outcomes standard. The quality of the research method was assessed using a tool that can assess the risks posed by each study design. A meta-analysis software program was used to calculate the mean effect size, effect size by intervention, and effect size by outcome. We also performed a meta-regression analysis and an analysis of publication bias.

Results: The mean effect size of the patients' gait was $0.65(p<0.05)$. The largest effect size by outcome was observed at the 6-m walk test, followed by the 6 -minutes walk test, 10 -m walk test, and the walking equipment test $(p<0.05)$. The meta-regression analysis showed that the effect size increased with increased duration, number, and length of sessions.

Conclusions: Aquatic exercise appears to show a moderate effect on the gait of chronic stroke survivors. Meta-analyses on the effects of aquatic exercise in other patient populations are needed. This study suggests standard criteria establishments for the effect of aquatic exercise on the walking ability of persons with chronic stroke.

Key Words: Hydrotherapy, Gait, Meta-analysis, Stroke

\section{Introduction}

Stroke survivors often experience loss of mobility and changes in consciousness levels and sensory functions due to neurological damage, and their functional movements are limited due to impaired cognitive and perceptual ability $[1,2]$. Unstable weight support due to asymmetric posture can have a great impact on the daily living and walking abilities of persons with stroke and may lead to secondary injuries, such as those from falls [3]. Improvement in the walking ability of persons affected by stroke is an important factor for independent living and is used as a measure of their recovery level $[4,5]$.
Aquatic exercise is effective in improving muscular strength, endurance, equilibrium ability, and cardiopulmonary endurance because it is less burdensome on the lower limbs [6,7]. It can provide stable training for those affected with stroke compared with ground exercises and can be helpful for achieving psychological stability [8]. Aquatic exercise is derived from aquatic therapy, which is a segment of orthopedic physical therapy, and has been developed and used as a strengthening program for the cardiovascular, neurological, and musculoskeletal systems in many countries [9]. It has been used in conjunction with conventional swimming, aquatic aerobics, walking using special floating devices, running, obstacle walking, aquatic obstacle training,

Received: 21 March, 2019 Revised: 7 May, 2019 Accepted: 21 May, 2019

Corresponding author: Sung-Hyoun Cho (ORCID https://orcid.org/0000-0002-5108-4342)

Department of Physical Therapy, Nambu University, 23 Cheomdanjungang-ro, Gwangsan-gu, Gwangju 62271, Republic of Korea Tel: 82-62-970-0232 Fax: 82-62-970-0492 E-mail: geriatricpt1@gmail.com

(c) This is an Open-Access article distributed under the terms of the Creative Commons Attribution Non-Commercial License (http://creativecommons.org/licenses/ by-nc/4.0) which permits unrestricted non-commercial use, distribution, and reproduction in any medium, provided the original work is properly cited.

Copyright $\odot 2019$ Korean Academy of Physical Therapy Rehabilitation Science 
and aquatic treadmill training [10,11]. In particular, the Halliwick method, Watsu method, and the Bad Ragaz ring method have attracted attention. These have been clinically applied using various aquatic treatment equipment in different medical and social welfare facilities [12].

Aquatic treadmill training significantly increased the walking field by $22 \%$, and many previous studies have reported an improved walking speed [13-15]. However, there were no inter- and intra-group differences in the findings of the $10-\mathrm{m}$ walk test in the aquatic and ground treadmill groups of a previous study [16]. Conversely, although there was a significant difference in the within-group comparison between the aquatic treatment group and Bobath approach group in another study, there was no between-group difference; this suggests that the difference in the effects varied according to the group [17]. Based on the results of these studies, it is necessary to perform a gait analysis and discuss and present the effects of aquatic exercise. However, no study has yet analyzed the effect size of aquatic exercise on the walking ability of stroke survivors in Korea.

As such, the purpose of this study was to present objective data on the effect of aquatic exercise on the gait of those affected with stroke by performing a meta-analysis (MA). This MA examined the effect of aquatic exercise on gait in comparison with the effects of other exercises performed underwater using individual effect sizes based on the results of studies that investigated aquatic exercise, aquatic obstacle training, aquatic rehabilitation, aquatic walking, aquatic treadmill training, and aquatic therapy for improving walking ability in persons with stroke.

\section{Methods}

\section{Search strategy}

Data research was conducted on electronic search sites, including Newnonmun (http://newnonmun.com), National Library (http://www.nl.go.kr), Korea Education and Research Information Service (KERIS) (http://www.riss.kr), Kyobo Scholar (http://scholar.kyobobook.co.kr), Academic Education Center (http://www.earticle.net), DBpia (http:// dbpia.com), KISS (http://kiss.ksudy.com), and NDSL (http:// scholar.ndsl.kr). The study was limited to domestic cases and aimed to minimize the heterogeneous context of domestic cases of stroke survivors on a clinical level from foreign cases and applied the outcome directly to domestic conditions. Therefore, this MA could be conducted in accordance with the international standards of relevance and con- sistency to which research should adhere to for scientific verification of the quality of the results [18]. This MA included studies that applied aquatic exercise in stroke survivors conducted between January 2006 and May 2017. The search strategy was a combination of MeSH (medical subject heading) terms and free text words including "hydrotherapy" [mesh], "stroke" [mesh], "hemiplegia" [mesh], "gait" [mesh], "hydrotherapy" [txt words], "stroke" [txt words], "hemiplegia" [txt words], and "gait" [txt words].

\section{Study selection}

This study analyzed the effects of domestic aquatic exercise on the gait of persons with stroke. This study was approved by the Institutional Review Board (IRB) of Nambu University, Gwangju, Republic of Korea (NBU-IRB-10414782017-HR-017). Since this study is a method to analyze existing research results, we received an expedited review with exemption from consent from the IRB committee. First, studies were searched on the basis of the participants, intervention, comparison, outcomes standard. Studies that conducted measurements before and after performing various aquatic methods for 4 to 12 weeks and presented the intervention effects with specific statistical values were selected. Studies with statistical values, such as mean, standard deviation, and sample number, which can be converted into effect sizes, were selected for the final analysis. Qualitative research, review articles, and studies with research designs that did not meet the criteria for this study were excluded. Studies that did not apply any intervention, those without effect size calculation, and poster research studies were excluded. To avoid excluding potentially relevant articles, the full-text paper was searched and examined when the abstract provided unclear information. Any disagreement was resolved through discussion. Full-text articles that satisfied the inclusion criteria were assessed by two reviewers with clinical knowledge of physical therapy and methodological knowledge of MA. References of the included articles were further checked manually.

\section{Data extraction}

In the first phase, the search was conducted using the keyword "hydrotherapy," which yielded 2,233 articles from the academic research information and 3,381 scholarly articles, resulting in a total of 5,614 articles. A total of 3,878 articles were found from the remaining search sites. Next, a total of 238 articles (120 journal articles and 118 academic articles with the combination of the keywords "stroke" and "hemi- 
plegia") were selected. In the second phase, 128 duplicate articles were eliminated, and the number was trimmed down to 110 articles. In the third phase, 92 articles were selected by removing 18 articles that included elderly subjects, children, animals, and individuals with disability. In the fourth phase, we excluded 32 articles that were not suitable for numerical error analysis and MA and extracted a total of 60 articles. In the last phase, 23 articles/studies that analyzed gait using walking equipment, the 6-m walk, $10-\mathrm{m}$ walk, and the 6-minutes walk tests were finally included; two professors read the full text of these articles for a thorough review.

\section{Quality assessment}

To improve the validity of the study results, the quality of the 23 final articles that met the selection criteria of this study was examined so that systematic errors did not result in or minimized the deviations in the results or estimates from the actual values and errors that could cause under- or overestimation of intervention effects [19]. For assessment, the risk of bias (RoB) tool was used for the randomized controlled trials (RCTs) and the risk of bias assessment tool for non-randomized studies (RoBANS) tool was employed for the non-randomized controlled trials (NRCTs) [18]. For RoB and RoBANS, the bias risk was divided into "high," "low," and "uncertain." A "low" bias risk indicates a low probability of bias; "high," high probability of bias; and "uncertain" indicates risk that is difficult to determine [18]. The quality of the selected articles was concluded after a rigorous review by two physiotherapy professors experienced in literature review and multiple meta-analyses when they both agreed on the quality [20].

\section{Data synthesis}

The Comprehensive Meta-Analysis software ver. 3.0 (Biostat Inc., Englewood, NJ, USA) was used for the effect size analysis. We extracted information on the characteristics of the participants and interventions in the 23 articles selected and coded the data according to the coding framework.

For all study results, the effect size was determined by calculating the Hedges's g, i.e., adjusted standardized mean difference considering the small sample sizes of the multiple studies. The $95 \%$ confidence interval (CI) was calculated, and the reciprocal value of the variance was used for the weight of each effect size. The random-effects model was applied for the mean effect sizes. To evaluate the statistical heterogeneity of the effect sizes, we first examined the forest plots. In addition, the overall observed variance, i.e., $\mathrm{Q}$ value, was calculated using the $\chi^{2}$ test; more specifically, the $\mathrm{I}^{2}$ value, which represents the actual variance and the interstudy variance for the overall observed variance, was calculated [20-23]. To test the moderator effect, a meta-ANOVA was performed for the categorical variable (effect according to the publication format) and a meta-regression analysis for the continuous variables (effect of the intervention period, number of interventions, and duration of each intervention) [19]. Finally, the validity of the study was verified by analyzing the publication bias for the validity of the entire study results.

\section{Results}

\section{Characteristics of the selected studies}

A total of 23 selected research articles were analyzed (Figure 1, Table 1) [14-17,24-41]. The data extracted consisted of the following: authors, year of publication, type of publication, type of research design, number of subjects, method of intervention, duration of application of intervention, number of applications, and measurement tools [19,42].

The publication year of the 23 articles in the final selection was ranged between 2006 to 2016; with the year 2010 as the reference point, 14 articles were published by 2010 and nine articles from 2011 to 2016. The articles analyzed included subjects with chronic stroke who had a cerebral infarction or a cerebral hemorrhage, and the subjects were assessed using gait training tools. Five articles had an experimental group only, and 18 articles had both experimental and control groups. Random and arbitrary classifications were conducted in 5 articles and 18 articles, respectively.

The analyses were conducted on a total of 435 subjects. There were 7 to 32 subjects in each article: nine articles had less than 20 subjects, twelve articles had 20-30 subjects, and two had 32 subjects. The types of exercise performed were aquatic task, aquatic rehabilitation, aquatic therapy, aquatic treadmill training, aquatic walking, and aquatic exercise. The application time of the interventions was 30-40 minutes in eight articles, 30 minutes in five articles, 40-60 minutes in 14 articles, and 70 minutes in one article. The number of interventions was 8-24 in eight articles, 30-36 in 13 articles, and 40-48 in two articles. The intervention period was 4 weeks in three articles, 6-10 weeks in seven articles, and 12 weeks in 13 articles. The assessment categories were com- 


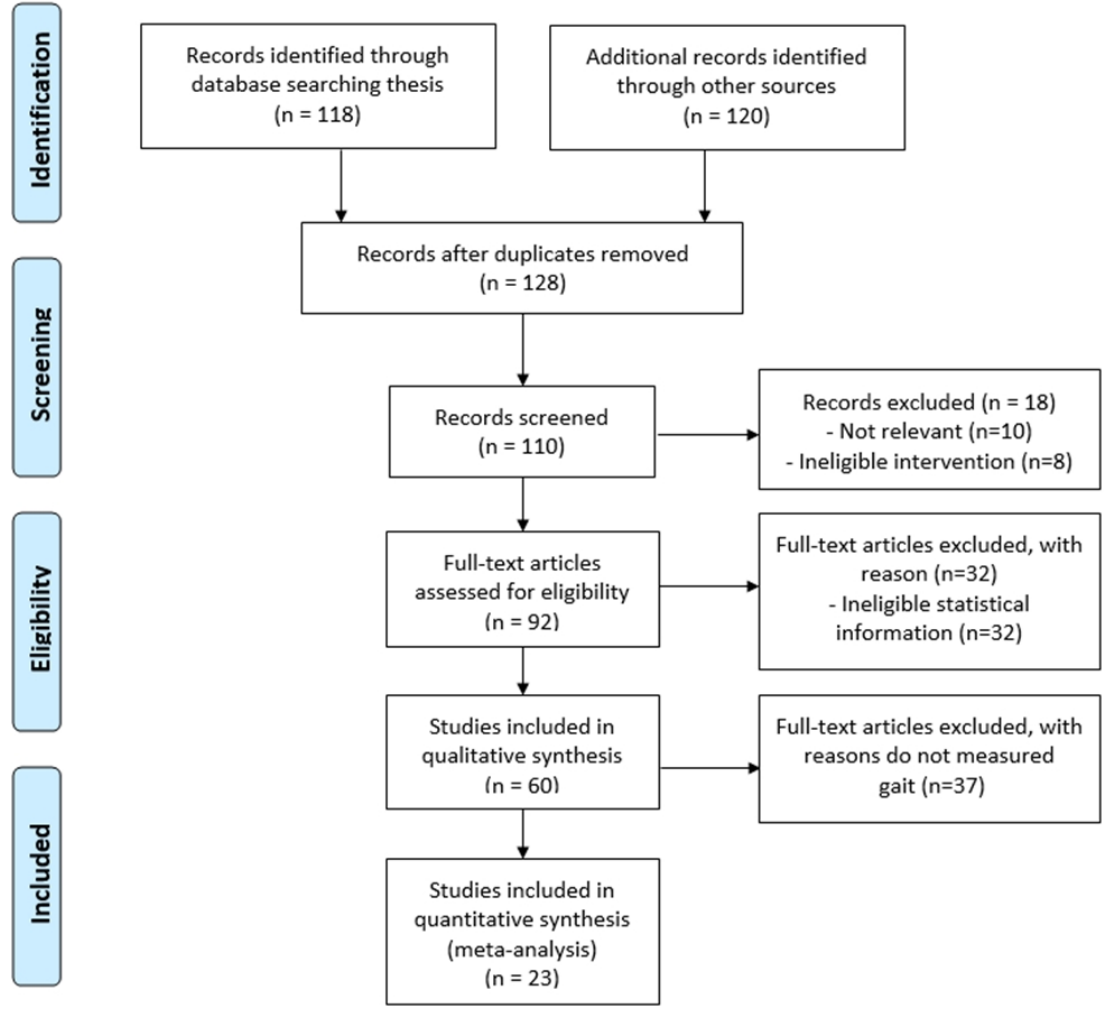

Figure 1. Flowchart of the study selection process.

Table 1. Characteristics of primary studies included in the analysis

\begin{tabular}{|c|c|c|c|c|c|c|c|c|c|c|c|c|c|}
\hline \multirow[b]{2}{*}{ First author, year } & \multirow[b]{2}{*}{ Effectsize } & \multirow[b]{2}{*}{$\begin{array}{l}\text { Publication } \\
\text { type }\end{array}$} & \multirow[b]{2}{*}{ RCT } & \multicolumn{10}{|c|}{ Aquatic exercise therapy } \\
\hline & & & & Intervention & Control & Intervention & $\begin{array}{c}\text { Duration } \\
\text { (wk) }\end{array}$ & $\begin{array}{l}\text { Session } \\
\text { number }\end{array}$ & $\begin{array}{l}\text { Length } \\
\text { (min) }\end{array}$ & $\begin{array}{l}\text { Walking } \\
\text { equipment }\end{array}$ & $\begin{array}{c}6-\mathrm{m} \\
\text { walk } \\
\text { test }\end{array}$ & $\begin{array}{c}10-\mathrm{m} \\
\text { walk } \\
\text { test }\end{array}$ & $\begin{array}{c}\text { 6-min } \\
\text { walk } \\
\text { test }\end{array}$ \\
\hline Cho and Cho, 2013 [27] & 2.40 & Journal & $x$ & 9 & 10 & Aquatic rehabilitation & 8 & 32 & 60 & & & & $\circ$ \\
\hline Cho and Jung, 2013 [24] & 1.51 & Journal & $\circ$ & 15 & 10 & Aquatic rehabilitation & 12 & 48 & 60 & & & & $\circ$ \\
\hline Chung, 2009 [28] & 0.50 & Thesis & $x$ & 5 & 5 & Aquatic exercise & 12 & 24 & 60 & $\circ$ & & & \\
\hline Kim, 2015 [25] & 0.19 & Thesis & $\circ$ & 10 & 9 & Aquatic gait training & 8 & 40 & 30 & $\circ$ & & & $\circ$ \\
\hline Kim, 2015 [14] & 2.26 & Thesis & $x$ & 12 & 10 & Aquatic rehabilitation & 12 & 36 & 70 & & $\circ$ & & \\
\hline Lee and Kang, 2010 [29] & 0.13 & Journal & $x$ & 8 & 8 & Aquatic gait training & 12 & 36 & 60 & & $\circ$ & & \\
\hline Lee and Kim, 2008 [30] & 0.77 & Journal & $x$ & 17 & 15 & Aquatic rehabilitation & 12 & 24 & 60 & & $\circ$ & & \\
\hline Lee and Lee, 2007 [31] & 0.69 & Journal & $x$ & 10 & 10 & Aquatic rehabilitation & 12 & 36 & 55 & & $\circ$ & & \\
\hline Lee, 2009 [32] & 1.32 & Thesis & $x$ & 17 & 15 & Aquatic task training & 12 & 36 & 50 & & & $\circ$ & \\
\hline Lee et al., 2009 [33] & 0.00 & Journal & $x$ & 10 & 10 & Aquatic gait training & 10 & 30 & 40 & $\circ$ & & & \\
\hline Lee et al., 2012 [34] & 0.16 & Journal & $x$ & 10 & 10 & Aquatic therapy & 6 & 30 & 40 & $\circ$ & & & \\
\hline Lee, $2011[35]$ & 0.47 & Thesis & $x$ & 10 & 10 & Aquatic rehabilitation & 12 & 36 & 60 & & $\circ$ & & \\
\hline Nam, 2006 [17] & 0.20 & Thesis & $x$ & 13 & 13 & Aquatic therapy & 6 & 18 & 40 & $\circ$ & & & \\
\hline Park, 2016 [36] & 1.01 & Thesis & $x$ & 13 & 15 & Aquatic exercise & 4 & 12 & 30 & $\circ$ & & & \\
\hline Park, 2010 [26] & 0.16 & Thesis & $\circ$ & 10 & 10 & Aquatic treadmill & 6 & 24 & 30 & $\circ$ & & & $\circ$ \\
\hline Park, 2010 [15] & 0.55 & Thesis & $\circ$ & 11 & 11 & Aquatic treadmill & 4 & 8 & 30 & $\circ$ & & & \\
\hline Yang, 2016 [16] & 0.03 & Thesis & $\circ$ & 10 & 10 & Aquatic treadmill & 4 & 12 & 30 & & & $\circ$ & \\
\hline Jeon, 2015 [37] & 0.42 & Thesis & $x$ & 20 & 0 & Aquatic rehabilitation & 12 & 36 & 60 & $\circ$ & & & \\
\hline Kim et al., 2006 [38] & 0.85 & Journal & $x$ & 7 & 0 & Aquatic rehabilitation & 12 & 36 & 50 & & & $\circ$ & \\
\hline Kim et al., 2006 [38] & 0.82 & Journal & $x$ & 7 & 0 & Aquatic rehabilitation & 12 & 36 & 50 & $\circ$ & & & \\
\hline Lee and Kang, 2009 [39] & 0.75 & Journal & $x$ & 8 & 10 & Aquatic rehabilitation & 12 & 24 & 60 & & & & $\circ$ \\
\hline Song and Kim, 2009 [40] & 0.23 & Journal & $x$ & 14 & 0 & Aquatic exercise & 10 & 30 & 60 & & & $\circ$ & \\
\hline Woo et al., 2007 [41] & 0.77 & Journal & $x$ & 7 & 0 & Aquatic rehabilitation & 12 & 36 & 50 & $\circ$ & & & \\
\hline
\end{tabular}

RCT: randomized controlled trial. 
posed of four variables total, which were measured in the walking equipment, 6-m walk, 10-m walk, and 6-minutes walk tests. Measurements were conducted on walking equipment in 11 articles. The speed obtained from the 6-m walking and $10-\mathrm{m}$ walking tests was measured in five and four articles, respectively, while the distance obtained from the 6-minutes walking test was assessed in five articles.

\section{Quality assessment of the selected studies}

The quality of the research method was assessed using a tool that can assess the risks posed by each study design. The RoB tool developed by the Cochrane group was used for the RCTs and the RoBANS tool for the NRCTs [18]. RoB as- sesses six areas: random assignment, sequencing concealment, participant and researcher blinding, blinding of the result evaluator, handling of incomplete results, and selective outcome reporting. RoBANS assesses subject group selection, confounding variables, intervention, blinding of assessment results, incomplete outcome data, and selective outcome reporting. For each item, the bias risk was classified into three categories: "low," which indicates a low probability of bias; "high," which indicates high probability of bias; and "uncertain," which indicates risk that is difficult to determine [18]. The results of the quality assessment of the 23 selected studies are shown in Tables 2 [15,16,24-26] and $3[14,17,27-41]$.

Table 2. Methodological evaluation of RCT study using RoB tool

\begin{tabular}{|c|c|c|c|c|c|c|}
\hline First author, year & $\begin{array}{c}\text { Random } \\
\text { sequence } \\
\text { generation }\end{array}$ & $\begin{array}{c}\text { Allocation } \\
\text { concealment }\end{array}$ & $\begin{array}{l}\text { Blinding of } \\
\text { participants } \\
\text { and persons }\end{array}$ & $\begin{array}{l}\text { Blinding of } \\
\text { outcome } \\
\text { assessment }\end{array}$ & $\begin{array}{l}\text { Incomplete } \\
\text { outcome }\end{array}$ & $\begin{array}{l}\text { Selective } \\
\text { reporting }\end{array}$ \\
\hline Cho and Jung, 2013 [24] & High & High & High & High & High & High \\
\hline Kim, 2015 [25] & High & High & High & High & High & High \\
\hline Park, 2010 [26] & High & High & High & High & High & High \\
\hline Park, 2010 [15] & High & High & High & Low & High & High \\
\hline Yang and Choi, 2016 [16] & Low & High & High & High & High & High \\
\hline
\end{tabular}

RCT: randomized controlled trial, RoB: risk of bias.

Table 3. Methodological evaluation of NRCT study using RoBANS tool

\begin{tabular}{|c|c|c|c|c|c|c|}
\hline First author, year & $\begin{array}{l}\text { Selection of } \\
\text { participants }\end{array}$ & $\begin{array}{c}\text { Confounding } \\
\text { variables }\end{array}$ & $\begin{array}{l}\text { Measurement of } \\
\text { intervention }\end{array}$ & $\begin{array}{l}\text { Blinding of } \\
\text { outcome } \\
\text { assessment }\end{array}$ & $\begin{array}{c}\text { Incomplete } \\
\text { outcome data }\end{array}$ & $\begin{array}{l}\text { Selective } \\
\text { reporting }\end{array}$ \\
\hline Cho and Cho, 2013 [27] & Low & High & High & High & Low & Low \\
\hline Chung, 2009 [28] & Low & High & High & High & Low & Low \\
\hline $\mathrm{Kim}, 2015$ [14] & Low & High & High & High & Low & Low \\
\hline Lee and Kang, 2010 [29] & Low & High & High & High & Low & Low \\
\hline Lee and Kim, 2008 [30] & Low & High & High & High & Low & Low \\
\hline Lee and Lee, 2007 [31] & Low & High & High & High & Low & Low \\
\hline Lee, 2009 [32] & Low & High & High & High & High & Low \\
\hline Lee et al., 2009 [33] & Low & High & High & High & Low & Low \\
\hline Lee et al., 2012 [34] & Low & High & High & High & Low & Low \\
\hline Lee, $2011[35]$ & Low & High & High & High & Low & Low \\
\hline Nam, 2006 [17] & Low & High & High & High & Low & Low \\
\hline Park, 2016 [36] & Low & High & High & High & Low & Low \\
\hline Jeon, 2015 [37] & Low & High & High & High & Low & Low \\
\hline Kim et al., 2006 [38] & Low & High & High & High & Low & Low \\
\hline Kim et al., 2006 [38] & Low & High & High & High & Low & Low \\
\hline Lee and Kang, 2009 [39] & Low & High & High & High & Low & Low \\
\hline Song and Kim, 2009 [40] & Low & High & High & High & Low & Low \\
\hline Woo et al., 2007 [41] & Low & High & High & High & Low & Low \\
\hline
\end{tabular}

NRCT: non-randomized controlled trial, RoBANS: risk of bias assessment tool for non-randomized studies. 


\section{Effect size of gait after aquatic exercise}

For the 23 selected studies, we used forest plots to present the results of calculating the standardized mean difference (i.e., Hedges's g) adjusted using the mean values of the preand post-intervention differences, standard deviation of the difference, and sample size of the experimental and control groups (Table 4). The mean effect size (Hedges's g) in all the studies was 0.65 (95\% CI, 0.43-0.87), indicating a medium effect size and statistical significance (Figure 2). When represented in terms of Cohen's [43] cumulative normalization distribution or $\mathrm{U} 3$, the mean value in the experimental group was equivalent to $\sim 73 \%$, while that in the control group was $50 \%$. When represented in terms of the binomial effect size display of Rosenthal and Rubin [44], which compares the success rates of the experimental and control groups, the success rate in the control group was $35 \%$, while that in the experimental group was $64 \%$. Therefore, the effect of aquat-

Table 4. Characteristics of sub-outcome variables for evaluation items for aquatic exercise

\begin{tabular}{|c|c|c|c|c|c|c|c|}
\hline \multirow{2}{*}{$\begin{array}{c}\text { Effect } \\
\text { variables }\end{array}$} & \multirow{2}{*}{$\mathrm{K}$} & \multirow{2}{*}{ Hedges's g } & \multicolumn{2}{|c|}{$95 \% \mathrm{CI}$} & \multicolumn{3}{|c|}{ Heterogeneity } \\
\hline & & & Lower & Upper & Q & $p$-value & $\mathrm{I}^{2}$ \\
\hline Total gait & 23 & 0.65 & 0.43 & 0.87 & 40.30 & 0.01 & 45.4 \\
\hline Walking equipment & 11 & 0.44 & 0.22 & 0.67 & 6.30 & 0.79 & 0.0 \\
\hline 6-m walk test & 5 & 0.82 & 0.21 & 1.44 & 10.08 & 0.04 & 60.3 \\
\hline 10 -m walk test & 4 & 0.59 & 0.02 & 1.16 & 7.76 & 0.05 & 61.3 \\
\hline 6-min test & 5 & 0.94 & 0.23 & 1.66 & 13.62 & 0.01 & 70.6 \\
\hline
\end{tabular}

$\mathrm{K}$ : number of effect size, CI: confidence interval, Q: total variability.

Model Study name

Outcome

\begin{tabular}{|c|c|c|c|c|c|c|}
\hline \multirow[b]{2}{*}{$\begin{array}{c}\text { Hedges's } \\
g\end{array}$} & & \multirow[b]{2}{*}{ Z-Value } & \multirow[b]{2}{*}{ p-Value } \\
\hline & $\begin{array}{l}\text { Standard } \\
\text { error }\end{array}$ & Variance & $\begin{array}{c}\text { Lower } \\
\text { limit }\end{array}$ & $\begin{array}{l}\text { Upper } \\
\text { limit }\end{array}$ & & \\
\hline 2.398 & 0.586 & 0.344 & 1249 & 3.547 & 4009 & 0.00 \\
\hline 1.505 & 0.470 & 0221 & 0.584 & 2.427 & 3201 & 0.00 \\
\hline 0.498 & 0.586 & 0.344 & -0.651 & 1.647 & 0.850 & 0.39 \\
\hline 0.192 & 0.440 & 0.194 & -0.671 & 1.054 & 0.435 & 0.66 \\
\hline 2.258 & 0.534 & 0286 & 1211 & 3.305 & 42266 & 0.0 \\
\hline 0.133 & 0.473 & 224 & -0.795 & 1.060 & 02200 & 0.77 \\
\hline 65 & 58 & 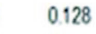 & 0.063 & 1.468 & 2.136 & 0.03 \\
\hline 0.692 & 0.442 & 95 & -0.175 & 1.558 & 1.564 & 0 \\
\hline 1.324 & 0.383 & 0.147 & 0.573 & 2074 & 3.457 & oc \\
\hline 0.000 & 0.428 & 0.183 & -0.839 & 0.839 & 0.000 & 10 \\
\hline 163 & 0.429 & 84 & -0.678 & 1.004 & 0.300 & 0. \\
\hline 0.467 & 0.435 & 0.189 & -0.385 & 1.318 & 1.073 & 02 \\
\hline 0.203 & 0.382 & 0.146 & -0.546 & 0.953 & 0.532 & 0 \\
\hline 1.010 & 0.403 & 0.163 & 0219 & 1.800 & 2.504 & oc \\
\hline 0.158 & 0.430 & 0.185 & .0685 & 1.000 & 0.367 & 0.1 \\
\hline 553 & 420 & 176 & -0.270 & 1.375 & 1.317 & 0 \\
\hline 033 & 28 & 83 & -0.806 & 0.873 & 0.078 & 09 \\
\hline 18 & 27 & 0.052 & -0.027 & 0.864 & 1.841 & $0 \mathrm{c}$ \\
\hline 0.848 & 0.399 & 0.159 & 0.066 & 1.631 & 2.124 & 0 \\
\hline 0.821 & 0.395 & & 0.047 & 1.596 & 2078 & 0 \\
\hline 0.750 & 0.334 & 0.112 & 0.095 & 1.405 & 2244 & 0 \\
\hline 0.227 & 0255 & 0065 & -0273 & 0.727 & 0890 & 0 \\
\hline 0.767 & 0.388 & 0.150 & 0.007 & 1.527 & 1.979 & 0 \\
\hline 06 & 0.081 & 0007 & 0.449 & 0.767 & 7.494 & 0 \\
\hline 850 & 0.113 & 0.013 & 0.428 & 0.872 & 5.744 & \\
\hline
\end{tabular}

\section{Hedges's $g$ and $95 \%$ a}

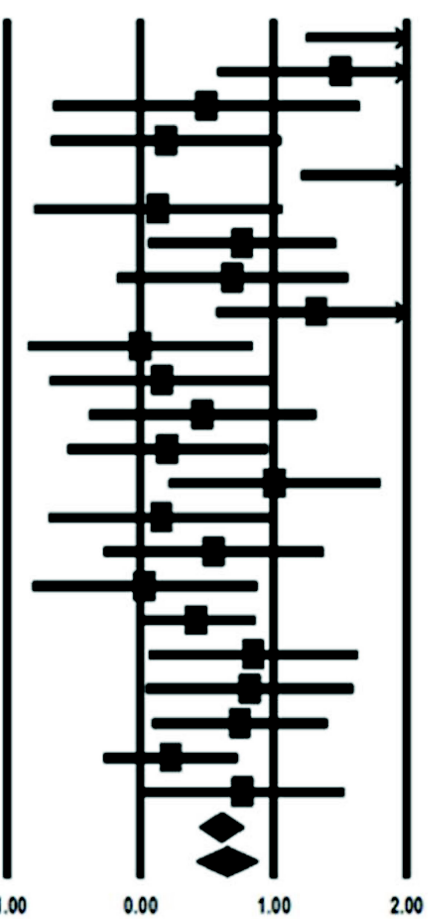

Favours A

Favours B

Figure 2. Forest plots for gait after aquatic exercise. 
ic exercise can be interpreted as moderate.

The overall aquatic walking effect size was Hedges's $\mathrm{g}=0.65(95 \% \mathrm{CI}, 0.43-0.87)$, and the effect was moderate. The heterogeneity or the percentage of variance across the studies in total variance was $\mathrm{I}^{2}=45.4 \%(\mathrm{Q}=40.30, p=0.01)$, indicating a moderate heterogeneity.

In the walking equipment test, the effect size was moderate, with Hedges's g=0.44 (95\% CI, 0.22-0.67), while no heterogeneity was found, with $\mathrm{I}^{2}=0 \%(\mathrm{Q}=6.30, p=0.79)$. In the 6-m walk test, the Hedges's g of 0.82 (95\% CI, 0.21 -

1.44) showed a large effect size, and the heterogeneity was moderate, with $\mathrm{I}^{2}=60.3 \%(\mathrm{Q}=10.08, p=0.04)$. The $10-\mathrm{m}$ walk test showed both moderate effect size and heterogeneity, with Hedges's g=0.59 (95\% CI, 0.02-1.16) and $\mathrm{I}^{2}=61.3 \%(\mathrm{Q}=7.76, p=0.05)$, respectively. The 6-minutes walk test showed a large effect size, with Hedges's g=0.94 (95\% CI, 0.23-1.66), and a moderate heterogeneity, with $\mathrm{I}^{2}=70.6 \%(\mathrm{Q}=13.62, p=0.01)$. The effect size in the 6-minutes walk test was the largest at 0.94 , followed by those in the $6-\mathrm{m}$ and $10-\mathrm{m}$ walk test and walking equipment test

(Figures 2-6).

\begin{tabular}{|c|c|c|c|c|c|c|c|c|c|}
\hline \multirow[t]{2}{*}{ Model } & \multirow[t]{2}{*}{ Study name } & \multirow[t]{2}{*}{ Outcome } & \multicolumn{7}{|c|}{ Statistics for each study } \\
\hline & & & $\begin{array}{l}\text { Hedges's } \\
\mathrm{g}\end{array}$ & $\begin{array}{l}\text { Standard } \\
\text { error }\end{array}$ & Variance & $\begin{array}{l}\text { Lower } \\
\text { limit }\end{array}$ & $\begin{array}{l}\text { Upper } \\
\text { limit }\end{array}$ & 2-Value & p-Valu \\
\hline & Kim KH, 2015 & $6 M$ & 2.258 & 0.534 & 0.286 & 1.211 & 3.305 & 4.226 & 0.0 \\
\hline & Lee YH, 2011 & $6 \mathrm{M}$ & 0.467 & 0.435 & 0.189 & -0.385 & 1.318 & 1.073 & 0.28 \\
\hline & Lee \& Kim, 2008 & $6 \mathrm{M}$ & 0.765 & 0.358 & 0.128 & 0.063 & 1.468 & 2.136 & 0.0 \\
\hline & Lee \& Kang, 2010 & $6 M$ & 0.133 & 0.473 & 0.224 & -0.795 & 1.060 & 0.280 & 0.7 \\
\hline & Lee \& Lee, 2007 & $6 \mathrm{M}$ & 0.692 & 0.442 & 0.195 & -0.175 & 1.558 & 1.564 & \\
\hline Fixed & & & 0.782 & 0.195 & 0.038 & 0.399 & 1.165 & 4.002 & \\
\hline ndom & & & 0.823 & 0.315 & 0.099 & 0.206 & 1.440 & 2.615 & \\
\hline
\end{tabular}

Model

Study name

Outcome

\section{$\mathrm{Kim} H J, 2015 \quad 6 \mathrm{~min}$}

Park SE, $2010 \quad 6$ min

Lee \& Kang, 2009 6min

Cho \& Cho, 2013 6min

Cho \& Jung, 2013 6min

Fixed

Random
Statistics for each study

\begin{tabular}{|c|c|c|c|c|c|c|}
\hline \multicolumn{7}{|c|}{ Statistics for each study } \\
\hline $\begin{array}{l}\text { Hedges's } \\
\quad \mathrm{g}\end{array}$ & $\begin{array}{l}\text { Standard } \\
\text { error }\end{array}$ & Variance & $\begin{array}{l}\text { Lower } \\
\text { limit }\end{array}$ & $\begin{array}{c}\text { Upper } \\
\text { limit }\end{array}$ & Z.Value & $p$-Value \\
\hline 0.267 & 0.441 & 0.195 & -0.597 & 1.132 & 0.606 & 0.545 \\
\hline 0.122 & 0.429 & 0.184 & -0.718 & 0.962 & 0.284 & 0.776 \\
\hline 0.750 & 0.334 & 0.112 & 0.095 & 1.405 & 2.244 & 0.025 \\
\hline 2.398 & 0.586 & 0.344 & 1.249 & 3.547 & 4.089 & 0.000 \\
\hline 1.505 & 0.470 & 0.221 & 0.584 & 2.427 & 3.201 & 0.001 \\
\hline 0.836 & 0.193 & 0.037 & 0.458 & 1.213 & 4.339 & 0.000 \\
\hline 0.942 & 0.365 & 0.133 & 0.227 & 1.656 & 2.583 & 0.010 \\
\hline
\end{tabular}

Hedges's $\mathrm{g}$ and $95 \% \mathrm{Cl}$

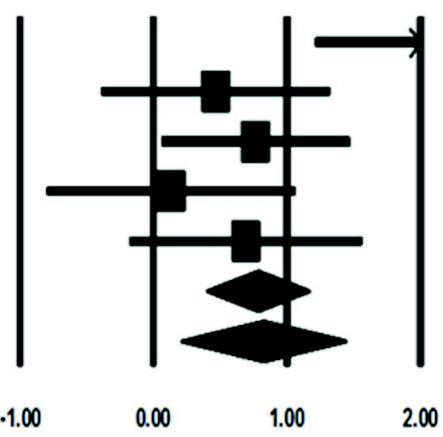

Favours A

Favours B
Hedges's g and $95 \% \mathrm{Cl}$

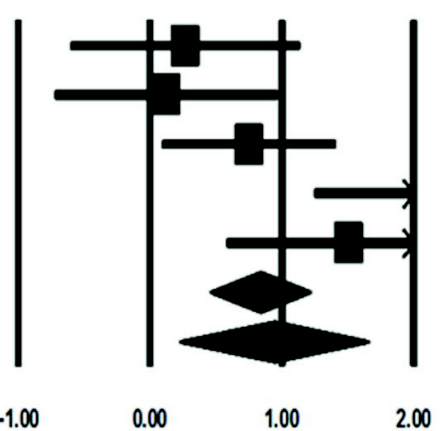

Figure 4. Forest plots for 6-m walk test after aquatic exercise. 


\begin{tabular}{|c|c|c|c|c|c|c|c|c|c|}
\hline \multirow[t]{2}{*}{ Model } & \multirow[t]{2}{*}{ Study name } & \multirow[t]{2}{*}{ Outcome } & \multirow[b]{2}{*}{$\begin{array}{c}\text { Hedges's } \\
\mathbf{g}\end{array}$} & \multirow[b]{2}{*}{$\begin{array}{l}\text { Standard } \\
\text { error }\end{array}$} & \multicolumn{3}{|c|}{ Statistics for each study } & \multirow[b]{2}{*}{ Z-Value } & \multirow[b]{2}{*}{ p-Value } \\
\hline & & & & & Variance & $\begin{array}{l}\text { Lower } \\
\text { limit }\end{array}$ & $\begin{array}{l}\text { Upper } \\
\text { limit }\end{array}$ & & \\
\hline & Lee DJ, 2009 & $10 \mathrm{M}$ & 1.324 & 0.383 & 0.147 & 0.573 & 2.074 & 3.457 & 0.001 \\
\hline & Yang HJ, 2016 & $10 \mathrm{M}$ & 0.033 & 0.428 & 0.183 & -0.806 & 0.873 & 0.078 & 0.938 \\
\hline & Kim KU et al, 2006 & $10 \mathrm{M}$ & 0.848 & 0.399 & 0.159 & 0.066 & 1.631 & 2.124 & 0.034 \\
\hline & Song \& Kim, 2009 & $10 \mathrm{M}$ & 0.220 & 0.255 & 0.065 & -0.280 & 0.719 & 0.862 & 0.389 \\
\hline Fixed & & & 0.528 & 0.172 & 0.029 & 0.191 & 0.864 & 3.075 & 0.002 \\
\hline Random & & & 0.589 & 0.289 & 0.084 & 0.023 & 1.155 & 2.038 & 0.042 \\
\hline
\end{tabular}

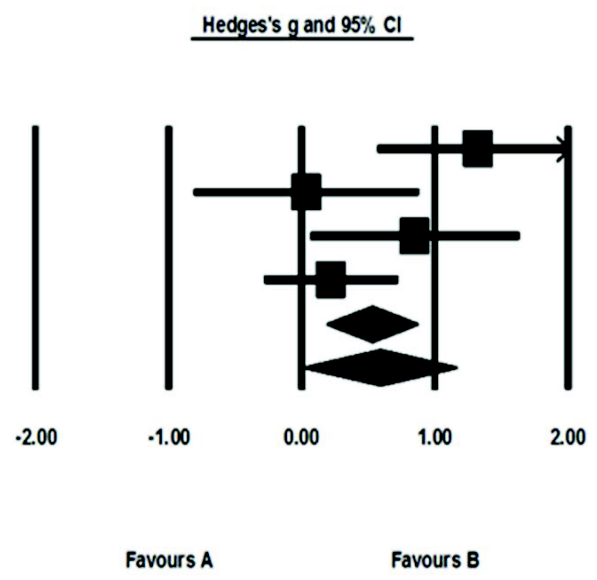

Figure 5. Forest plots for 10-m walk test after aquatic exercise.

\begin{tabular}{|c|c|c|c|c|c|c|c|c|c|}
\hline \multirow[t]{2}{*}{ Model } & \multirow[t]{2}{*}{ Study name } & \multirow[t]{2}{*}{ Outcome } & \multicolumn{7}{|c|}{ Statistics for each study } \\
\hline & & & $\begin{array}{l}\text { Hedges's } \\
\mathbf{g}\end{array}$ & $\begin{array}{c}\text { Standard } \\
\text { error }\end{array}$ & Variance & $\begin{array}{l}\text { Lower } \\
\text { limit }\end{array}$ & $\begin{array}{l}\text { Upper } \\
\text { limit }\end{array}$ & $z \cdot$ Value & p.Value \\
\hline & Chung HY, 2009 & Combined & 0.498 & 0.586 & 0.344 & -0.651 & 1.647 & 0.850 & 0.396 \\
\hline & Jeon IG, 2011 & Combined & 0.418 & 0.227 & 0.052 & -0.027 & 0.864 & 1.841 & 0.066 \\
\hline & Kim HJ, 2015 & Combined & 0.173 & 0.440 & 0.194 & -0.690 & 1.035 & 0.393 & 0.695 \\
\hline & Kim KU et al, 2006b & stride length & 0.821 & 0.395 & 0.156 & 0.047 & 1.596 & 2.078 & 0.038 \\
\hline & Lee SY et al, 2009 & gaitvelocity & 0.000 & 0.428 & 0.183 & -0.839 & 0.839 & 0.000 & 1.000 \\
\hline & Lee TH et al, 2012 & gait velocity & 0.163 & 0.429 & 0.184 & -0.678 & 1.004 & 0.380 & 0.704 \\
\hline & $\mathrm{Nam} \mathrm{HC}, 2006$ & Combined & 0.219 & 0.383 & 0.147 & -0.531 & 0.969 & 0.572 & 0.568 \\
\hline & Park BS, 2016 & Combined & 1.010 & 0.403 & 0.163 & 0.219 & 1.800 & 2.504 & 0.012 \\
\hline & Park SE, 2010 & Combined & 0.170 & 0.430 & 0.185 & -0.673 & 1.013 & 0.394 & 0.693 \\
\hline & Park SW, 2010 & Combined & 0.553 & 0.420 & 0.176 & -0.270 & 1.375 & 1.317 & 0.188 \\
\hline & Wo SY et al, 2007 & Combined & 0.767 & 0.388 & 0.150 & 0.007 & 1.527 & 1.979 & 0.048 \\
\hline Fixed & & & 0.443 & 0.115 & 0.013 & 0.218 & 0.669 & 3.852 & 0.000 \\
\hline Random & & & 0.443 & 0.115 & 0.013 & 0.218 & 0.669 & 3.852 & 0.000 \\
\hline
\end{tabular}

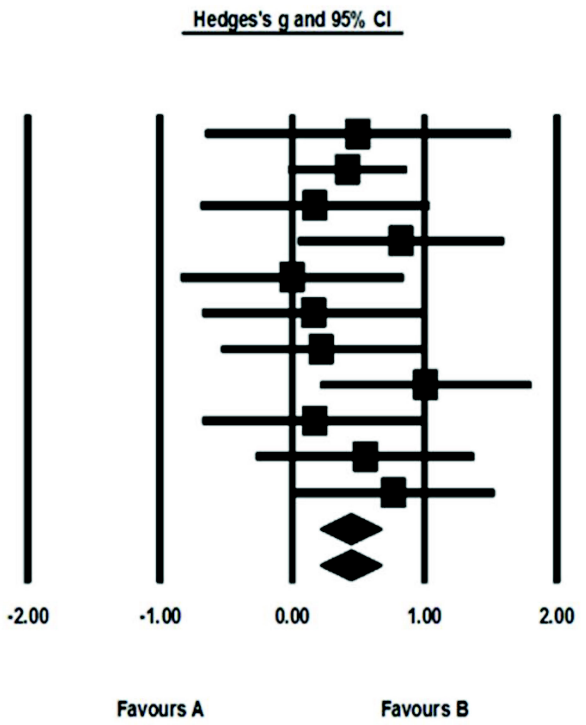

Figure 6. Forest plots for 6-minutes walk test after aquatic exercise.

Heterogeneity test for the effect size: analysis of moderating effects

The overall mean effect size was found to be Hedges's $\mathrm{g}=0.65$ (95\% CI, 0.43-0.87), and the heterogeneity was moderate $(25 \%-75 \%)$ as suggested by Higgins and Green [18] $\left(\mathrm{I}^{2}=45.4 \%, \mathrm{Q}=40.30, p=0.01\right)$. Therefore, it was decided that exploratory explanations on the background of effect size heterogeneity and a meta-ANOVA was conducted as in previous studies using the study design and publication type as moderators [19,20].

The effect size in the NRCT group was 0.70 , and that in the RCT group was 0.47 , showing a larger effect size in the former. However, the $\mathrm{Q}_{\mathrm{b}}$ value between the groups was 0.64 ( $\mathrm{df}=1, p=0.42)$, showing no significant difference. In the analysis of the difference in the effect size between the thesis papers and journal articles, the effect size in the former was 0.61 , and that in the latter was 0.69 , showing a larger effect size for the journal articles. However, the $Q_{b}$ value between the groups was $0.12(\mathrm{df}=1, p=0.73)$, and there was no difference in the effect size between them (Table 5).

In addition, a meta-regression analysis was conducted to determine the heterogeneity in the effect sizes using the continuous variables (duration, number, and length of sessions of aquatic exercise) as the moderating variables.

The regression analysis for the duration of sessions 
Table 5. Meta-ANOVA by study design and publication type

\begin{tabular}{|c|c|c|c|c|c|c|c|c|}
\hline \multirow{2}{*}{ Category } & \multirow{2}{*}{ Subgroup } & \multirow{2}{*}{$\mathrm{K}$} & \multirow{2}{*}{ Hedges's g } & \multicolumn{2}{|c|}{$95 \% \mathrm{CI}$} & \multirow{2}{*}{$Z(p)$} & \multirow{2}{*}{$\mathrm{I}^{2}(\%)$} & \multirow{2}{*}{$\mathrm{Q}_{\mathrm{b}}(p)$} \\
\hline & & & & Lower limit & Upper limit & & & \\
\hline \multirow[t]{2}{*}{ Study design } & NRCT & 18 & 0.70 & 0.45 & 0.95 & $5.43(<0.01)$ & 48.1 & $0.64(0.42)$ \\
\hline & $\mathrm{RCT}$ & 5 & 0.47 & -0.03 & 0.97 & $1.83(>0.05)$ & 41.6 & \\
\hline \multirow[t]{2}{*}{ Publication type } & Thesis & 11 & 0.61 & 0.28 & 0.94 & $3.61(<0.01)$ & 49.3 & $0.12(0.73)$ \\
\hline & Journal & 12 & 0.69 & 0.38 & 1.00 & $4.32(<0.01)$ & 46.2 & \\
\hline
\end{tabular}

$\mathrm{K}$ : number of effect size, CI: confidence interval, $\mathrm{Q}_{\mathrm{b}}$ : Q value (between the two subgroups), NRCT: non-randomized controlled trial, RCT: randomized controlled trial.
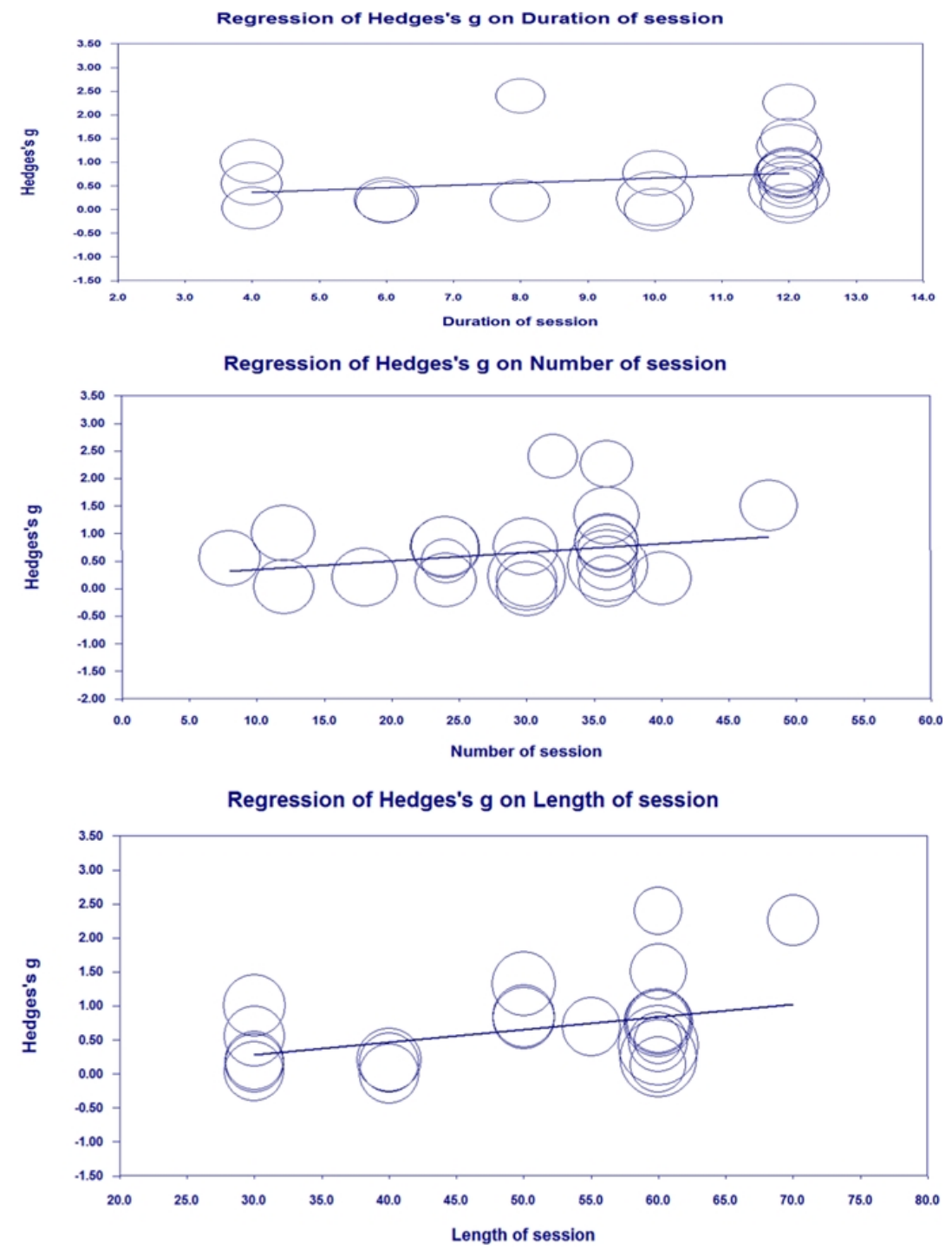

Figure 7. Regression analysis of Hedges's $g$ by duration of session and number of session and length of session.

showed that the longer the duration of the sessions, the larger the effect size; however, this was not significant $(Z=1.37$; $95 \%$ CI, $-0.02-0.13$ ). Conversely, the regression analysis for the number of sessions showed that the effect size increased as the number of sessions increased; however, this finding was also not significant $(Z=1.32 ; 95 \% \mathrm{CI},-0.01$ 0.04). The regression analysis for the length of sessions showed that the effect size increased as the length of the sessions increased ( $Z=2.11 ; 95 \%$ CI, 0.00-0.04) (Figure 7). 


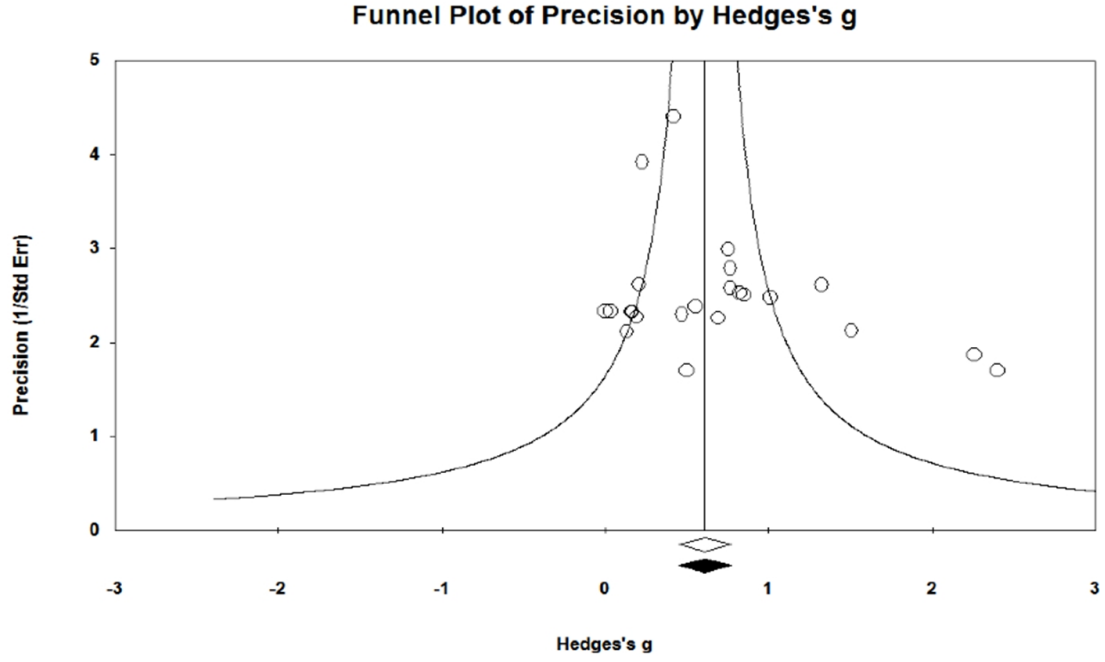

Figure 8. Funnel plot of precision by Hedges's g.

\section{Analysis of publication bias}

To verify the validity of the results, the publication bias among the 23 articles was analyzed. An error was identified by analyzing the funnel plots [22]. As shown in Figure 8, the effect size was not visually symmetrical. For objective verification, Egger's regression test [45], which is a statistical analysis method for publication errors, was used to examine the relationship between the effect size and the standard error.

The value for bias was $2.16(\mathrm{t}=1.88, \mathrm{df}=21, p>0.01)$, and the statistical analysis showed a publication error, but with no significance. In the analysis of the fail-safe $\mathrm{N}$ using the conventional method, the safety factor value was 346 , demonstrating reliability. Finally, when publication bias was suspected, it was re-analyzed using the Duval and Tweedie's trim-and-fill method [46]. When the trim-and-fill method was applied, there was no newly filled effect size, and the adjusted and observed mean effect sizes were all 0.65 , showing no difference. In addition, the $95 \% \mathrm{CI}$ of the adjusted mean effect size was $0.43-0.87$, which was significant.

\section{Discussion}

Meta-analyses can provide information for establishing a rational and valid basis for clinical decision-making, avoiding unnecessary repetitive research, and finding comprehensive results that can be generalized by integrating and analyzing individual research results systematically [19]. After excluding articles according to the criteria, 11 thesis papers and 12 journal articles were included in this MA (analyzing a total of 23 studies). The subjects in these articles were persons with chronic stroke who had cerebral infarction or cerebral hemorrhage. Aquatic exercise provides stability and load during walking in patients via water buoyancy, hydrostatic pressure, and resistance [33]. Based on this evidence, we examined the total effect size and effect sizes of the variables, including the findings of the walking equipment test and the $6-\mathrm{m}, 10-\mathrm{m}$, and 6 -minutes walk test.

The analysis of the gait results obtained after aquatic exercise in the stroke survivors showed that the mean effect size was moderate, with a Hedges's g of 0.65 (95\% CI, $0.43-0.87)$. These results indicate that aquatic exercise has a positive effect on walking ability of persons with stroke. The largest effect size was observed in the 6-minutes walk test (0.94), followed by the 6 -m walk test (0.82), 10-m walk test (0.59), and walking equipment test (0.44). As such, aquatic therapy has at least a moderate positive effect on the walking ability of those with chronic stroke. The 6-minutes walk test is assumed to have shown the largest effect size as it reflects the exercise capacity required for daily living and the response of all systems related to gait for the assessment of exercise capacity in the elderly and in patients with peripheral vascular disease [47].

In the experimental group wherein aquatic walking exercise was conducted in persons with hemiplegia, the 6-m walk, timed up and go test, and 12-minutes walk test results significantly improved compared with those before the intervention [29]. In addition, the application of the 12-week aquatic rehabilitation exercise program in persons with hemiplegia after stroke showed a significant difference in the 6-minutes walk test depending on the group and the 
measurement timing, as well as the interaction effect between the groups and measurement timing [24].

The RS-scan system was used to evaluate the stability, foot pressure, and walking speed during stance in those with hemiplegia. Those who underwent a 10 -week aquatic walking exercise showed significant increases in pressure in the big toe, heel, and metatarsal areas, with decreased motion of the subtalar joint and a significant decrease in the foot angle. In addition, the walking speed also significantly increased [33]. When the Smart Step System was used to measure the walking speed of the patients, the walking speed improved in the aquatic therapy group [35]. These results show that aquatic exercise can have a positive effect on the walking ability of stroke survivors.

As a limitation of the present study, there was difficulty in conducting this MA owing to the apparent lack of studies conducted on aquatic exercise only; most conventional physiotherapy studies also included basic physiotherapy in addition to the major intervention given the ethical issues with persons with stroke. In addition, there were difficulties in classifying the variables as the same dependent variables or assessments were sometimes differently categorized in each study. If there are various independent and dependent variables that are difficult to include in a meta-analyses, it is necessary to perform the analyses separately and review the results; it is believed that the combination of significance tests and index estimates of effect sizes can overcome these difficulties. Future research should include overseas papers on aquatic exercise to reduce the reporting bias [48,49]. In future randomized trials, the categories of experimental variables should be clearly classified, and related guidelines should be established.

Nevertheless, this study may be able to suggest standard criteria for the effect of aquatic exercise on the walking ability of persons with chronic stroke and help other researchers in selecting aquatic exercise methods and measurement tools in the future.

\section{Acknowledgements}

This work was supported by a National Research Foundation of Korea grant funded by the Korea government (MSIT) (No. 2017R1C1B5076499).

\section{Conflict of Interest}

The authors declared no potential conflicts of interest with respect to the authorship and/or publication of this article.

\section{References}

1. Kolb B, Gibb R. Brain plasticity and recovery from early cortical injury. Dev Psychobiol 2007;49:107-18.

2. Grotta JC, Welch KM, Fagan SC, Lu M, Frankel MR, Brott T, et al. Clinical deterioration following improvement in the NINDS rt-PA Stroke Trial. Stroke 2001;32:661-8.

3. Orlin MN, McPoil TG. Plantar pressure assessment. Phys Ther 2000;80:399-409.

4. Salbach NM, Mayo NE, Robichaud-Ekstrand S, Hanley JA, Richards CL, Wood-Dauphinee S. The effect of a task-oriented walking intervention on improving balance self-efficacy poststroke: a randomized, controlled trial. J Am Geriatr Soc 2005; 53:576-82.

5. Kim CM, Eng JJ. The relationship of lower-extremity muscle torque to locomotor performance in people with stroke. Phys Ther 2003;83:49-57.

6. Noh DK, Lim JY, Shin HI, Paik NJ. The effect of aquatic therapy on postural balance and muscle strength in stroke survivors/a randomized controlled pilot trial. Clin Rehabil 2008;22:966-76.

7. Park JH, Chung YJ. The effects of an additional weight aquatic exercise program on balance and lower extremity strength in persons with stroke: randomized controlled study. Phys Ther Rehabil Sci 2018;7:6-12.

8. Matsumoto I, Araki H, Tsuda K, Odajima H, Nishima S, Higaki $\mathrm{Y}$, et al. Effects of swimming training on aerobic capacity and exercise induced bronchoconstriction in children with bronchial asthma. Thorax 1999;54:196-201.

9. Lee DJ. Effect of the aquatic training on balance and walking in stroke patients. J Spec Educ Rehabil Sci 2008;47:21-41.

10. Kim EH. Principles and effects of rhythmic and aquatic exercises. J Muscle Jt Health 1998;5:296-302.

11. Han SK. Changes in leg range of motion and muscle activity of chronic stroke patient during obstacle tasking on the ground and aquatic [Doctoral thesis]. Daejeon: Eulji University; 2014.

12. Park GD, Kang SH. The effect of aquatic-rehabilitation program to 40-age CVA patients' flexibility and walking time. J Sport Leis Stud 2007;29:375-85.

13. Masumoto K, Shono T, Takasugi S, Hotta N, Fujishima K, Iwamoto Y. Age-related differences in muscle activity, stride frequency and heart rate response during walking in water. $\mathrm{J}$ Electromyogr Kinesiol 2007;17:596-604.

14. Kim KH. Effects of an aquatic rehabilitation exercise program on daily living fitness, blood lipid, balance, and walking ability in patients with hemiplegia after stroke [Doctoral thesis]. Gwangju: Chosun University; 2015.

15. Park SW. The effect of under-water treadmill gait training on gait and balance ability in patients with chronic stroke [Master thesis]. Seoul: Sahmyook University; 2010.

16. Yang HY, Choi JD. Effects of underwater treadmill gait training on gait, balance, and pulmonary function in stroke patients. Phys Ther Korea 2015;22:34-43.

17. Nam HC. Effect of aquatic therapy for functional restoration in the persons with chronic stroke [Doctoral thesis]. Daegu: Daegu 
University; 2006.

18. Higgins JPT, Green S. Cochrane handbook for systematic reviews of interventions. Chichester: John Wiley \& Sons; 2008.

19. Park WJ, Park SJ, Hwang SD. Effects of cognitive behavioral therapy on attention deficit hyperactivity disorder among schoolaged children in Korea: a meta-analysis. J Korean Acad Nurs 2015;45:169-82.

20. Han MH, Lee KH. Effects of communication ability enhancement program for nursing students in Korea: a systematic review and meta-analysis. J Korean Acad Soc Nurs Educ 2017;23:1526.

21. Kim EY, Hwang SD, Kim EJ. Stimulation-oriented interventions for behavioral problems among people with dementia: a systematic review and meta-analysis. J Korean Acad Nurs 2016;46: 475-89.

22. Borenstein M, Hedges LV, Higgins JPT, Rothstein HR. Introduction to meta-analysis. Chichester: John Wiley \& Sons; 2009.

23. Kim AR, Yang IS. Effects of exercise on endothelial progenitor cells in cardiovascular disease patients: a systematic review. J Korea Acad Ind Coop Soc 2017;18:366-79.

24. Cho WJ, Jung HY. Effects of the aquatic rehabilitation exercise program on physical fitness and blood lipid in stroke hemiplegic. Korean J Sports Sci 2013;22:973-83.

25. Kim HJ. Effects of progressive aquatic gait training for balance, gait and endurance in patient with stroke [Master thesis]. Seoul: Sahmyook University; 2015.

26. Park SE. Comparison of effect of aquatic and land treadmill exercise on the stroke patient's gait and physical function [Master thesis]. Yongin: Yongin University; 2010.

27. Cho WJ, Cho HC. Effects of 8-week aqua therapy and treadmill walking exercise on daily physical fitness and inflammatory markers in stroke hemiplegia patients. J Sport Leis Stud 2013;52: 851-60.

28. Chung HY. The effect of water-based exercise program on gait coordination for people in chronic stroke [Master thesis]. Seoul: Seoul National University; 2009.

29. Lee YH, Kang SJ. The effects of 12-week aquatic gait and land exercises on gait capacity in hemiplegia disable. Korean J Phys Educ 2010;49:457-64.

30. Lee YH, Kim JH. The effects of aquatic rehabilitation exercise on relation factors of gait in hemiplegic male disabled after CVA. Korean Soc Adapt Phys Act Exerc 2008;16:39-54.

31. Lee YH, Lee HK. Effect of aquatic rehabilitation exercise on the maximal walking velocity and gait endurance in man patients with hemiplegia after stroke. J Coach Dev 2007;9:83-91.

32. Lee DJ. A study on effect of task-related training in water and on land for chronic stroke patient for a balance, functional performance and the quality of the life [Doctoral thesis]. Seoul: Sahmyook University; 2009.

33. Lee SY, Hyong IH, Shim JM. The effect of aquatic gait training on foot kinesiology and gait speed in right hemiplegic patients. J
Korea Contents Assoc 2009;9:674-82.

34. Lee TH, Kim SY, Kim JP, Kang EC, Lee SP. The effect of therapeutic intervention by aquatic physical therapy on stroke patient. J Korean Acad Clin Electrophysiol 2012;10:15-21.

35. Lee YH. The effects of detraining and retraining by exercise rehabilitation types on gait capacity and recurrent associated factors of ischemic stroke in hemiplegia disabled with ischemic stroke [Doctoral thesis]. Seoul: SangMyung University; 2011.

36. Park BS. Change of spatiotemporal gait parameters in post stroke patients according to various therapeutic exercises [Doctoral thesis]. Yongin: Yongin University; 2016.

37. Jeong GH. The effects of upper-lower functional training in underwater on balance and pulmonary function of chronic stroke [Doctoral thesis]. Daegu: Daegu University; 2015.

38. Kim KU, Kim HM, Woo SY, Chung BK. The effect of the aqua-rehabilitation program on the stroke patient's muscle strength and ADL performance. J Adap Phys Activ 2006;14:99115 .

39. Lee YH, Kang SJ. Effects of aquatic rehabilitation exercise with land rehabilitation exercise on physical fitness for activities of daily living and range of motion of low limbs in chronic hemiplegia. J Adap Phys Activ 2009;17:121-41.

40. Song JM, Kim SM. The effect of aquatic exercise on the improvement of physical and pulmonary function after stroke. J Korean Soc Phys Ther 2009;21:15-22.

41. Woo SY, Kang DH, Kim HJ. A gait pattern analysis of the stroke patients using the aqua rehabilitation program. Soonchunhyang J Nat Sci 2007;13:165-70.

42. Shin HH, Shin YH, Kim GE. Effect of cognitive behavioral therapy (CBT) for perinatal depression: a systematic review and meta-analysis. J Korea Acad Ind Coop Soc 2016;17:271-84.

43. Cohen J. Statistical power analysis for the behavioral sciences. 2nd ed. Hillsdale (NJ): Lawrence Erlbaum Associates; 1998.

44. Rosenthal R, Rubin DB. A simple, general purpose display of magnitude of experimental effect. J Educ Psychol 1982;74:166-9.

45. Egger M, Davey Smith G, Schneider M, Minder C. Bias in meta-analysis detected by a simple, graphical test. BMJ 1997; 315:629-34.

46. Duval S, Tweedie R. A nonparametric "trim and fill" method of accounting for publication bias in meta-analysis. J Am Stat Assoc 2000;95:89-98.

47. Enright PL. The six-minute walk test. Respir Care 2003;48:7835.

48. Hwang SJ, Woo YK. Fall prevention strategies in community-dwelling older adults aged 65 or over with type 2 diabetes mellitus: a systematic review and meta-analysis. Phys Ther Rehabil Sci 2018;7:197-203.

49. Lee DS, Hwang SJ. Motor imagery on upper extremity function for persons with stroke: a systematic review and meta-analysis. Phys Ther Rehabil Sci 2019;8:52-59. 